\title{
Development of a Method for the Synthesis of Adaptive Spectral Operators for the Analysis of Random Signals
}

\author{
Abdenbi Abenaou
}

\begin{abstract}
This article suggests an approach for the synthesis of adaptive spectral operators from the general spectral kernel. This method provides an infinite set of configurable basis functions which fulfill the fundamental criterion of the orthonormality and the completeness of system functions to ensure a spectral analysis with a fast calculation algorithm. Relying on this approach, its implementation leads to solving the problem of random signals compression.
\end{abstract}

Index Terms-Spectral orthogonal operator, fast transformation, spectral kernels.

\section{INTRODUCTION}

In signal processing, the first idea that comes to mind is the minimization of space description of data to analyze. To deal with such problems, conventional methods based on spectral transformation in the Fourier, Walsh and Haar's function are often used for their fast algorithm. Though put into practice these transformations do not always lead to a considerable compression of data to analyze. Thus, their implementation is limited in case of real random signals.

Minimizing the data description space can be achieved through applying the Karhunen-Loeve transformation [1]. However, its practical use is limited due to the difficulty of calculating its own functions, and the absence of a fast transform algorithm for computing the spectrum.

Therefore, handling this problem requires the synthesis of a linear spectral system with configurable basis functions that will ensure an adaptable rapid transformation of signals analysis. To this end, this article suggests a method based on the spectral representation of the operator using the general spectral kernel. This method consists of the decomposition of the matrix operator as the product of sparse matrices whose non-zero elements are interconnected according to the condition of the orthonormality and the completeness, and having a proportion of zeros providing parametric building of basis functions with a fast transform algorithm.

\section{A METHOD FOR REPRESENTING THE TRANSFORM} OPERATOR USING THE GENERALIZED SPECTRAL KERNEL

In digital signal processing, the orthogonal linear transform of a signal $X$ can be represented by the matrix equation:

Manuscript received June 1, 2016; revised December 6, 2016.

Abdenbi Abenaou is with Ibn Zohr University, National School of Applied Sciences -Agadir, Morocco (e-mail: a.abenaou@uiz.ac.ma).

$$
Y=\frac{1}{N} H X
$$

where:

$-X=\left[x_{1}, x_{2}, \ldots, x_{N}\right]^{\mathrm{T}}$ is the original signal to transform having the size $N=2^{\mathrm{n}}$;

$-Y=\left[y_{1}, y_{2}, \ldots, y_{N}\right]^{\mathrm{T}}$ is the vector of spectral coefficients calculated by the orthogonal spectral operator $H$ of dimension $N$ x $N$.

The factorization of Good [2], has demonstrated a possibility for representing the matrix operator $H$ as the product of sparse matrices $G_{\mathrm{i}}(2)$ with a proportion of higher zeros which allows the construction of fast Fourier, Haar and Walsh's transform algorithm. The matrix $G_{\mathrm{i}}(i=1, \ldots, n)$ has the following form:

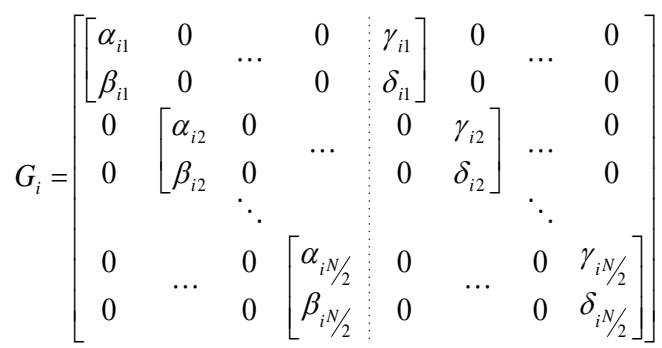

Using the binary notation of rows and column numbers $(z$ $=z_{\mathrm{n}} z_{\mathrm{n}-1} \ldots z_{1}$ and $\left.u=u_{\mathrm{n}} u_{\mathrm{n}-1} \ldots u_{1}\right)$, and considering the particular case $(N=8)$, the matrix $G_{\mathrm{i}}$ can be represented as follows:

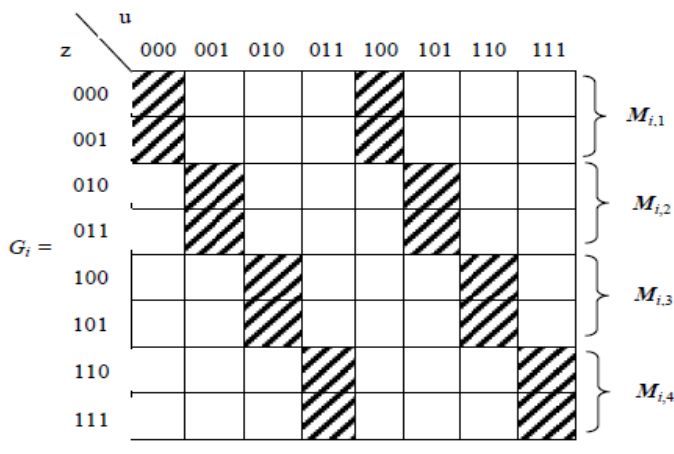

Fig.1. Representation of the structure of the good's matrix.

with: $M_{i, j}=\left\lfloor\begin{array}{lllll}\alpha_{00}^{i, j} & 0 & 0 & 0 & \alpha_{01}^{i, j} \\ \alpha_{10}^{i, j} & 0 & 0 & 0 & \alpha_{11}^{i, j}\end{array}\right\rfloor$

The white and black squares represent the zero and nonzero values of the $G_{i}$ matrix. We can see that the non-zero elements of the two adjacent rows are building on micro-matrix or blocks of matrix $M_{i, j}(i=1, \ldots n ; j=1, \ldots N / 2)$. The non-zero elements of blocks form the matrix $V_{i, j}$ having a minimum dimension that will be called later spectral kernels.

To satisfy the two basis requirements of the orthonormality 
and the completeness of the basis functions, the general spectral kernel takes the following form:

$$
\begin{aligned}
& V_{i, j}=\left\lfloor\begin{array}{ll}
\alpha_{00}^{(i, j)} & \alpha_{01}^{(i, j)} \\
\alpha_{10}^{(i, j)} & \alpha_{11}^{(i, j)}
\end{array}\right\rfloor=\sqrt{2}\left[\begin{array}{cc}
\cos \left(\varphi_{i, j}\right) & w_{i, j} \sin \left(\varphi_{i, j}\right) \\
\sin \left(\varphi_{i, j}\right) & -w_{i, j} \cos \left(\varphi_{i, j}\right)
\end{array}\right\rfloor \\
& w_{i, j}=\exp (j \theta), \quad \varphi \in\left[\begin{array}{ll}
0, & 2 \pi
\end{array}\right], \quad \theta \in\left[\begin{array}{ll}
0, & 2 \pi
\end{array}\right]
\end{aligned}
$$

where the parameters $\varphi_{i, j}$ and $\theta_{i, j}$ can take random values which influence the proportion of zeros of matrix $G_{i}$. This makes it possible to synthesize an infinite set of the basis functions having a fast transform algorithm, including Fourier, Walsh and Haar.

To evaluate the spectral kernels' parameters $\varphi_{i, j}$ and $\theta_{i, j}$ of matrix $G_{i}$, we can form the spectral operators $H$ with complex basis functions and when $\theta_{i, j}=0$, the real operators depending on the terms of the problem to deal with.

For this reason, the relation (3) is called the general spectral kernel. The total number of the kernels of the formed spectral operator is equal to $n * N / 2$, which allows to obtain a very important proportion of zeros that equals $n^{*} N$.

The spectral kernels may be different, partially or completely identical. For example, Walsh-Hadamard's basis functions [3]-[7] are formed from identical kernels with spectral parameters $\left(\theta_{i, j}=0\right.$, et $\left.\varphi_{i, j}=\varphi_{j}=\frac{\pi}{4}\right)$, and Haar's basis functions [4], [6], [8] are formed with the parameters $\left(\varphi_{1}=\frac{\pi}{2}, \varphi_{2}=0\right)$, while those of Fourier are formed by the kernels such $\left(\varphi_{i, j}=\frac{\pi}{4}, \quad \theta_{i, j}=(i-1) \frac{\pi}{N}\right)$.

For basis functions synthesized from identical kernels (such as Walsh's) spectral operator thus obtained can be calculated by the Kronecker's product [9] of spectral kernels. Usually, for a high proportion of zeros, the spectral operator cannot be calculated in such a way. In this article, to synthesize a large class of orthogonal basis functions, we suggest to use the Kronecker's modified product as follows.

\section{Synthesis OF THE SPeCtRAL Operator Using THE KRONECKER MODIFIED PRODUCT}

The class of synthesized basis functions may be extended if the matrix used instead of the Kronecker's product is made according to the rule:

$$
A \otimes B=\left\lfloor\begin{array}{ll}
a_{00}^{1} & a_{01}^{1} \\
a_{10}^{1} & a_{11}^{1}
\end{array}\right\rfloor \otimes B=\left\lfloor\begin{array}{cc}
a_{00}^{1} B & a_{01}^{1} B \\
a_{10}^{1} B & a_{11}^{1} B
\end{array}\right\rfloor
$$

We use the modified form:

$$
\begin{aligned}
& \left\lfloor\begin{array}{ll}
a_{00}^{1} & a_{01}^{1} \\
a_{10}^{1} & a_{11}^{1}
\end{array}\right\rfloor \otimes\left\lfloor\begin{array}{l}
B^{1} \\
B^{2}
\end{array}\right\rfloor=\left\lfloor\begin{array}{ll}
a_{00}^{1} B^{1} & a_{01}^{1} B^{1} \\
a_{10}^{1} B^{2} & a_{11}^{1} B^{2}
\end{array}\right\rfloor \\
& =\left[\begin{array}{cccc}
a_{00}^{1} b_{00}^{1} & a_{00}^{1} b_{01}^{1} & a_{01}^{1} b_{00}^{1} & a_{01}^{1} b_{01}^{1} \\
a_{00}^{1} b_{10}^{1} & a_{00}^{1} b_{11}^{1} & a_{01}^{1} b_{10}^{1} & a_{01}^{1} b_{11}^{1} \\
a_{10}^{1} b_{00}^{2} & a_{10}^{1} b_{01}^{2} & a_{11}^{1} b_{00}^{2} & a_{11}^{1} b_{01}^{2} \\
a_{10}^{1} b_{10}^{2} & a_{10}^{1} b_{11}^{2} & a_{11}^{1} b_{10}^{2} & a_{11}^{1} b_{11}^{2}
\end{array}\right]
\end{aligned}
$$

We can observe that the second multiplying equation (5) has the aspect of a matrix column formed by two spectral kernels. The next multiplier can be composed of four different kernels, and so on. In this case the shape of the Kronecker's modified product corresponds to the following expression:

$$
[A] \otimes\left[\begin{array}{ll}
B^{(1)} & B^{(2)}
\end{array}\right]^{T} \otimes\left[\begin{array}{llll}
C^{(1)} & C^{(2)} & C^{(3)} & C^{(4)}
\end{array}\right]^{T}
$$

The number of spectral kernels for each following multiplier increases by 2 times. We must note that this multiplication rule is unchangeable to the left of matrix A whatever the size $N=2^{n}$. Based on this multiplication rule mentioned above and using the relation (6), the spectral operator $H$ can be expressed as follows:

$$
\begin{aligned}
& H=\left[V_{n, 1}\right] \otimes\left[\begin{array}{ll}
V_{n-1,1} & V_{n-1,2}
\end{array}\right]^{T} \otimes \ldots \otimes\left[\begin{array}{llllll}
V_{i, 1} & V_{i, 2} & \ldots & V_{i, l} & \ldots & V_{i, 2^{n-1}}
\end{array}\right]^{T} \otimes \ldots \\
& \ldots \otimes\left[\begin{array}{llllll}
V_{1,1} & V_{1,2} & \ldots & V_{1, l} & \ldots & V_{1,2^{2-1}}
\end{array}\right]^{T}, \\
& H=\prod_{i=1}^{n} G_{n-i+1}=\otimes \prod_{i=1}^{n}\left[V_{i, 1}, \ldots, V_{i, k}, \ldots V_{i, N / 2}\right]
\end{aligned}
$$

where $V_{i, j}$ are the spectral kernels.

Using the equation (8) we calculate the spectral operator for $N=8$. Thus, the corresponding kernels are:

$$
\begin{gathered}
V_{1, j}=C^{(j)}=\left[c_{z_{i} u_{i}}^{(j)}\right], \quad V_{2, j}=B^{(j)}=\left[b_{z_{i} u_{i}}^{(j)}\right], \\
V_{3, j}=A^{(j)}=\left[a_{z_{i} u_{i}}^{(j)}\right]
\end{gathered}
$$

From (7):

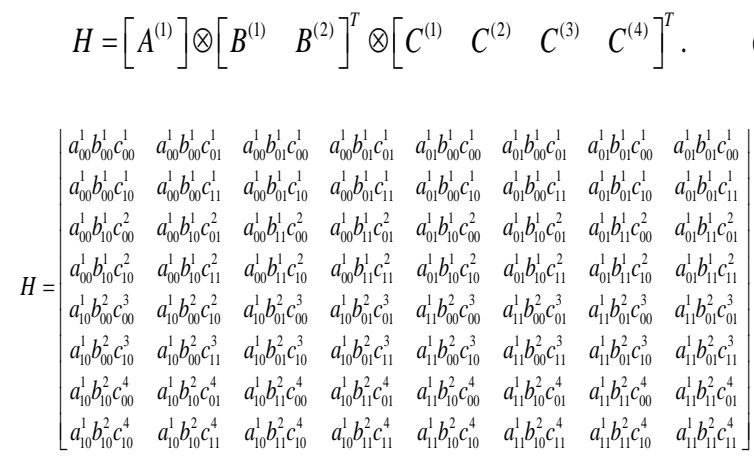

Fig. 2. Calculation of the spectral operator

Analyzing the elements $h_{z, u}$ of the operator $H$ (Fig. 2), we can notice a regularity of repetition of the elements $a, b$ and $c$. The recurrence of these elements allows the extraction from the global matrix of the blocks with the dimension respectively $N / 2 \times N / 2, N / 4 \times N / 4$ and $N / 8 \times N / 8$.

The representation of the regularity of the elements $h_{z, u}$ allows to obtain a topological scheme that can be generalized for any size $N=2^{n}$ of the operator $H$. The $h_{z, u}$ elements of the operator $H$ can then be calculated using the relation:

$$
\begin{gathered}
h_{z, u}=\prod_{i=1}^{n} \alpha_{z_{i} u}^{(i, j)} \\
\left(j=1+z_{i+1} \cdot 2^{0}+z_{i+2} \cdot 2^{1}+\ldots+z_{n} \cdot 2^{n-2}\right)
\end{gathered}
$$

For example the element $h_{5,6}$ from the operator $H$ of Figure 3 can be calculated using equation (11) as follows: For all $i=$ $1,2,3$ we have $: h_{z, u}=h_{5,6}=h_{101,110}$ then $z=z_{3} z_{2} z_{1}=101$ 
and $u=u_{3} u_{2} u_{1}=110$

Considering (9) and (11):

$$
\alpha_{z_{1} u_{1}}^{(1,3)}=\alpha_{10}^{(1,3)}=c_{10}^{(3)}
$$$$
\alpha_{z_{2} u_{2}}^{(2,2)}=\alpha_{01}^{(2,2)}=b_{01}^{(2)}
$$

$\alpha_{z_{3} u_{3}}^{(3,1)}=\alpha_{11}^{(3,1)}=a_{11}^{(1)}$

whence: $h_{101,110}=a_{11}^{(1)} b_{01}^{(2)} c_{10}^{(3)}$

that is conform to the topological scheme of the Fig. 3.

The expression of the operator $\mathrm{H}$ using the modified Kronecker's product significantly increases the classes of synthesized basis functions without complicating the procedure of obtaining the operator's elements using the Kronecker normal product.

An important property of the representation of this topological scheme consists of a possibility to act locally on the synthesized operator via the change of some concrete elements of the spectral kernel. This property is useful when setting the basis functions of the spectral operator [10] (particularly in some rows or columns of the matrix operator $\mathrm{H})$ depending on the conditions of the problem to deal with, without disturbing the orthogonality and completeness of system functions.

\section{Determination of Angular Parameters of the SPECTRAL KERNELS}

Whether $X$ is a standard from a recording of some signals represented by a vector sized $N=2^{n}$ :

$$
X=\left[x_{1}, x_{2}, \ldots, x_{N}\right]^{T}
$$

whose spectral representation may be obtained by:

$$
Y=\frac{1}{N} H X
$$

and expressed with a single non-zero spectral component such as:

$$
Y=\left[y_{1}, 0, \ldots, 0\right]^{T} \quad \text { avec } y_{1} \neq 0
$$

Using the equation (7), we can represent the spectral operator $\mathrm{H}$ as a real matrix whose spectral kernels express the following:

$$
V_{i, j}=\sqrt{2}\left[\begin{array}{cc}
\cos \left(\theta_{i, j}\right) & \sin \left(\theta_{i, j}\right) \\
\sin \left(\theta_{i, j}\right) & -\cos \left(\theta_{i, j}\right)
\end{array}\right\rfloor
$$

that can be considered as a particular case of the general spectral kernels (3).

Thus, from (13) we obtain the transposed vector of the signal $X$ :

$$
X^{T}=N H^{T} Y
$$

For example, if $N=8$, the spectral operator can be calculated as follows:

$$
\begin{aligned}
H= & \sqrt{8}\left[\left(\begin{array}{cc}
c_{11} & s_{11} \\
s_{11} & -c_{11}
\end{array}\right),\left(\begin{array}{cc}
c_{12} & s_{12} \\
s_{12} & -c_{12}
\end{array}\right),\left(\begin{array}{cc}
c_{13} & s_{13} \\
s_{13} & -c_{13}
\end{array}\right),\left(\begin{array}{cc}
c_{14} & s_{14} \\
s_{14} & -c_{14}
\end{array}\right)\right] \otimes \ldots \\
& {\left[\left(\begin{array}{cc}
c_{21} & s_{21} \\
s_{21} & -c_{21}
\end{array}\right),\left(\begin{array}{cc}
c_{22} & s_{22} \\
s_{22} & -c_{22}
\end{array}\right),\left(\begin{array}{cc}
c_{23} & s_{23} \\
s_{23} & -c_{23}
\end{array}\right),\left(\begin{array}{cc}
c_{24} & s_{24} \\
s_{24} & -c_{24}
\end{array}\right)\right] \otimes \ldots } \\
& \otimes\left[\left(\begin{array}{cc}
c_{31} & s_{21} \\
s_{31} & -c_{21}
\end{array}\right),\left(\begin{array}{cc}
c_{32} & s_{32} \\
s_{32} & -c_{32}
\end{array}\right),\left(\begin{array}{cc}
c_{33} & s_{33} \\
s_{33} & -c_{33}
\end{array}\right),\left(\begin{array}{cc}
c_{34} & s_{34} \\
s_{34} & -c_{34}
\end{array}\right)\right]
\end{aligned}
$$

where $c_{i j}=\cos \left(\theta_{i j}\right)$ and $s_{i j}=\sin \left(\theta_{i j}\right)$.

Multiplying $H^{T}$ by the column vector $Y$ whose elements are zero except for the first, we obtains the vector $X^{T}$, composed by the product of coefficient $y_{l}$ by the elements of the first column of $H^{T}$

$$
\left.\mid \begin{array}{l}
x_{1} \\
x_{2} \\
x_{3} \\
x_{4} \\
x_{5} \\
x_{6} \\
x_{7} \\
x_{8}
\end{array}\right\rfloor=8 \sqrt{8}\left|\begin{array}{l}
y_{1} c_{11} c_{21} c_{31} \\
y_{1} c_{11} c_{21} s_{31} \\
y_{1} c_{11} s_{21} c_{32} \\
y_{1} c_{11} s_{21} s_{32} \\
y_{1} s_{11} c_{22} c_{33} \\
y_{1} s_{11} c_{22} s_{33} \\
y_{1} s_{11} s_{22} c_{34} \\
y_{1} s_{11} s_{22} s_{34}
\end{array}\right|
$$

The relation (18) builds a system of equations with the variables $y_{1}, \theta_{1}, \theta_{2}, \ldots, \theta_{7}$ which we can compute the parameters $\theta_{\mathrm{i}}$ with the following operations:

$$
\begin{gathered}
\operatorname{tg}\left(\theta_{21}\right)=\sqrt{\frac{\left(x_{3}^{2}+x_{4}^{2}\right)}{\left(x_{1}^{2}+x_{2}^{2}\right)}} \quad \operatorname{tg}\left(\theta_{22}\right)=\sqrt{\frac{\left(x_{7}^{2}+x_{8}^{2}\right)}{\left(x_{5}^{2}+x_{6}^{2}\right)}} \\
\operatorname{tg}\left(\theta_{11}\right)=\sqrt{\frac{\left(x_{5}^{2}+x_{6}^{2}+x_{7}^{2}+x_{8}^{2}\right)}{\left(x_{1}^{2}+x_{2}^{2}+x_{3}^{2}+x_{4}^{2}\right)}}
\end{gathered}
$$

Based on this calculation procedure we can find a general form to determine $\theta_{\mathrm{i}}$ parameters of the spectral operator $H$ for any size $N=2^{n}$. With the parameter values found $\theta_{\mathrm{i}}$, the synthesis of the operator $H$, and its representation in the form of a product was performed according to the topological scheme described above. Thus, we have to bear in mind that if we increase the size of the initial data, the parameters of the kernels of the operator previously obtained do not change. The elements of the new spectral cores will be defined by the supplementary coefficients of the signal.

In the general case, we can calculate the angular parameters of the kernels by the relation:

$$
\operatorname{tg}\left(\theta_{i, j}\right)=\frac{x_{2 j}}{x_{2 j-1}} \operatorname{tg}\left(\theta_{1, j}\right)=\sqrt{\frac{\sum_{i=\frac{N}{2}+1}^{N} x_{i}^{2}}{\sum_{i=1}^{\frac{N}{2}} x_{i}^{2}}}
$$

The coefficient $y_{1}$ decomposition can be determined by the Parseval's relation:

$$
y_{1}^{2}=\frac{1}{N} \sum_{i=1}^{N} x_{i}^{2}
$$

The proposed method for synthesis of adaptive basis functions can be used in various fields of signal processing such as technical diagnosis [10]. In [10] a setting procedure and an adaptation of basis functions elaborated with a fast transform algorithm. In Fig. 3 are shown the experimental results obtained from the comparison between the spectrums of a vibration signal Fig. $3 \mathrm{~b}$ calculated using the adaptive basis functions and those obtained using conventional basis 
functions, those of Fourier, Walsh and Haar (Fig. 3c, Fig. 3d and Fig. 3e). According to the aspect of spectrum obtained using the adaptive basis function, it can be noticed that a rapid decay of the components which may promote a better compression of the signal to be analyzed in comparison with conventional methods.

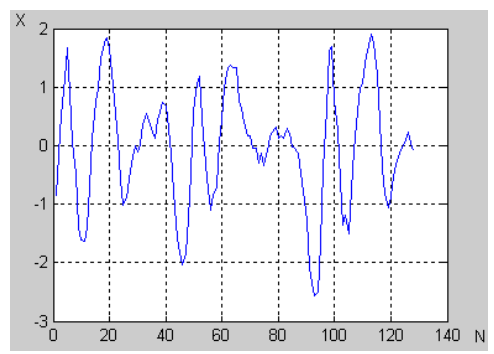

a. Fragment of a recording signal to be analyzed

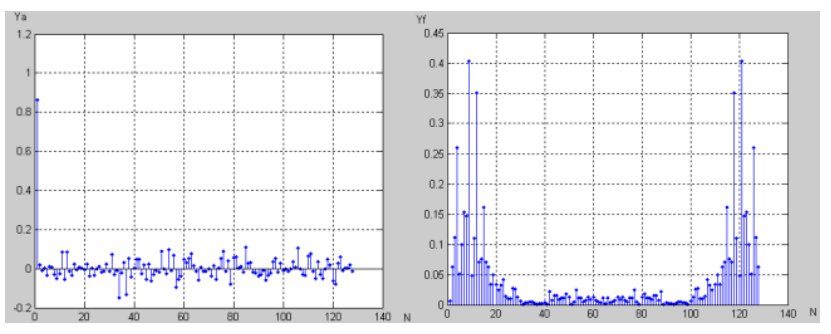

b. Adaptive spectrum

c. Fourier spectrum

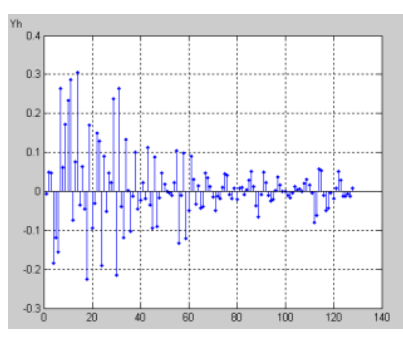

d. Walsh spectrum

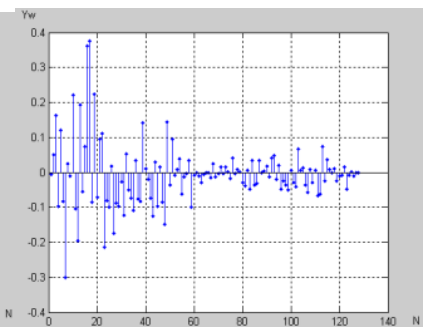

e. Haar spectrum

Fig.3. Comparison between the spectrum of the signal obtained using the adaptive basis function and that obtained by conventional basis (Walsh, Haar, Fourier).

\section{CONCLUSION}

The expression of the operator with spectral processing kernels allows to create a fast algorithm, with which the passage from a basis function to another is only using some modification of the parameters of kernels, which gives a possibility not only to synthesize a wide class of function systems but to form bases with desired properties and predict the peculiarity of their behavior us well.

The problem dealt with in this article reflects a temptation to find a constructive approach to the synthesis of basis functions with a fast algorithm and their practical use in signal processing problems.

Moreover, the suggested approach includes in a single mathematical model, conventional basis functions of systems such as Fourier, Walsh and Haar, discovered at different times, and gives the right to form an infinite variety of new functions with rapid transformation algorithm bases.

The settings of represented spectral operators based on spectral kernels gives opportunities to optimize the basis functions to solve the space minimization problem of signals' description and extraction of informative features which is important to the resolution of various signal processing problems such as the diagnosis of dynamic systems and signal recognition.

\section{REFERENCES}

[1] A. Isar, "Andrei cubitchi miranda nafornita, algorithmes et techniques de compression," Editura Orizonturi Politehnice, 2002.

[2] I. J. Good, "The interaction algorithm and practical fourier analysis," $J$. Roy. Statist. Soc. Ser. B, B-20, pp. 361-372, 1958.

[3] R. Rolland, "Remarques sur le calcul de la transformation d'Hadamard, " Dossiers Acrypta, 2008.

[4] Master-Siao. [Online]. Available: http://master-siao.enseeiht.fr/documents/doc_cours/basesfonction.pdf

[5] H. B. Kekre, S. Thepade, and A. Maloo, "Performance comparison of image retrieval using fractional coefficients of transformed image using DCT, Walsh, Haar and Kekre's transform", CSC-International Journal of Image Processing, vol. 4, no. 2, pp. 142-155, May 2010.

[6] H. B. Kekre, T. Sarode, S. Natu, and P. Natu, "Performance comparison of speaker identification using DCT, Walsh, Haar On full and row mean of spectrogram," International Journal of Computer Applications, August 2010

[7] G. Lazaridis and M. Petrou, "Image registration using the Walsh transform," IEEE Trans. Image Process, vol. 15, no. 8, pp. 2343-2357, 2006.

[8] M. Petrou and P. G. Sevilla, Image Processing: Dealing with Texture, New York: Wiley, 2006.

[9] M. A. Thornton, D. M. Miller, and R. Drechsler, Transformations amongst the Walsh, Haar, Arithmetic and Reed-Muller Spectral Domains.

[10] A. Abdenbi, "Méthode et algorithme de classification des signaux à base des transformations orthogonales adaptables - Application pour les systèmes de diagnostique," $1^{\text {èr }}$ JDTIC'09, ENSIAS, Rabat Maroc, 2009.

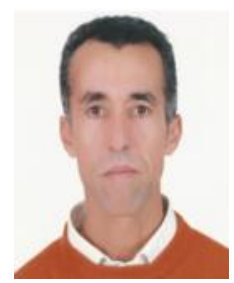

Abdenbi Abenaou was born in 1967 in Casablanca Morocco. He graduated from The Bonch-Bruevich St Petersburg, State University of Telecommunications in 1998.

He obtained his Ph.D degrees in technical sciences from the St. Petersburg State Electrotechnical University "LETI" in 2005. Now he is a professor of the Department of Software Engineering at the National School of Applied Sciences-Agadir, Morocco. His research interests are methods and algorithms for the recognition of signals and images. 